\title{
HABILITACIÓN DE UNA CIUDAD EN SUCESOS CON MÚLTIPLES VÍCTIMAS
}

\section{HABILITATION OF A CITY IN CASE WITH MULTIPLE VICTIMS}

Nogué Navarro L. ${ }^{a}$; Nociarová D. ${ }^{b, c}$; Bardalet Viñals $N .^{a-d}$; Hospital Ribas A. ${ }^{a-d}$; Adserias Garriga, MJ. ${ }^{a-\mathrm{e}}$

${ }^{a}$ Facultad de Medicina, Universitat de Girona, ${ }^{b}$ Universitat Autònoma de Barcelona, ${ }^{c}$ Visualforensics, ${ }^{d}$ Instituto de Medicina Legal de Cataluña, división Girona, ${ }^{e}$ Área de Ciencias Forenses, Universidad Rey Juan Carlos, Madrid.

Corresponding autor: Nogué Navarro L.

laia_nogue@hotmail.com

Telf.: (+34) 619631130

Fax: (+34) 972419617

Número de palabras del manuscrito: 1323 


\section{HABILITACIÓN DE UNA CIUDAD EN SUCESOS CON VÍCTIMAS MÚLTIPLES}

\section{Introducción}

En los últimos años se ha dado un incremento de los accidentes con víctimas múltiples, debido a los avances en las comunicaciones y los transportes. En España se han dado diferentes accidentes aéreos y ferroviarios en distintos puntos del país. Por este motivo, se cree conveniente que cada ciudad tenga un plan de gestión de catástrofes con una serie de espacios habilitados para poder responder en caso de un accidente de estas características. En catástrofes anteriores se ha habilitado espacios como pabellones feriales y deportivos al presentar un amplio espacio con electricidad, refrigeración y agua corriente, siendo muy adecuado para acoger a los cadáveres, restos y objetos personales, así como realizar las labores de identificación ${ }^{1-2}$.

El objetivo de este artículo es presentar la habilitación de espacios aplicable en cualquier capital de provincia de España para la respuesta postmortem ante una catástrofe, tomando como ejemplo la ciudad de Girona.

Se ha tomado esta ciudad como modelo por presentar infraestructuras viarias, ferroviarias, portuarias y aeroportuarias cada vez más concurridas.

Se espera, que este artículo sea de gran ayuda para los lectores y puedan aplicarlo a sus ciudades ante la respuesta post mortem a una catástrofe.

\section{Estudio de las necesidades para dar respuesta al suceso}

Es de suma importancia conocer con antelación las características, geográficas y demográficas, así como los medios materiales que se disponen antes de habilitar espacios ${ }^{3}$. Para ello, se ha establecido un plan de gestión de cadáveres en la ciudad de Girona, en una supuesta catástrofe. Se ha tenido en cuenta todos los espacios disponibles de la ciudad para ubicar a los cadáveres. El Instituto de Medicina Legal y la Facultad de Medicina de la Universidad de Girona (UdG) para el suministro del material, y el Palacio de Justicia de Girona, para ubicar el Centro de Integración de Datos e acogida de familiares ${ }^{3-4}$.

Para poder confeccionar el plan, se consultó a los siguientes cargos:

- Alcaldía de Girona y presidencia del Palau Firal.

- Jefatura de protección Civil del Ayuntamiento de Girona.

- Jefatura de Bomberos de la Región de Emergencias de Girona.

- Subdirección de la sala mando de los Mossos d'Esquadra de Girona.

- Jefatura de la Policía Municipal de Girona.

- Dirección Territorial de Emergencias Médicas de Girona.

- Jefatura de la Unidad Territorial de Policía científica de Girona.

- Subdirección de la División de la Policía Científica. Cos dels Mossos d'Esquadra.

- Dirección Grupo MÉMORA Girona.

- Delegación de Grupo MÉMORA a la zona este.

- Dirección de la zona logística Grupo MÉMORA Girona.

- Decanato de la Facultad de Medicina de la Universitat de Girona. Dirección del Centro de Genética Cardiovascular UdG-IDIBGI (Institut d'Investigació Biomèdica de Girona). Cardiólogo del Hospital Universitario Dr. Josep Trueta. Dirección del Postgrado de Medicina Forense. Identificación de Cadáveres y Actuación en Grandes Catástrofes. 


\section{Espacios habilitados}

1. Ubicación del Centro de Integración De Datos y acogida de los familiares de las víctimas mortales ${ }^{3-4}$ :

Tras un desastre con un número significativo de cadáveres, identificación y así poder entregar los restos humanos correspondientes a sus familiares.

Aprovechando que el Palau de Justícia está justo delante del Palau Firal 100m, y 900m del Pabelló Municipal Fontajau, el edificio se habilitaría como punto de Centro de Integración de Datos y de acogida a los familiares de las víctimas mortales.

Distribución del edificio:

- Los Jueces que están de guardia, en los despachos de ellos

- Planta baja: Sala de prensa

- $1^{\text {a }}$ Planta: Zona de recogida de datos antemortem

- $1^{\text {a }}$ Planta: Zona de comparación de datos antemortem postmortem

- $3^{\text {a }}$ Planta: Donde se localiza el Instituto de Medicina Legal de la división de Girona, la sala psicológica y psiquiátrica para acoger a los familiares

2. Habilitación de espacios para la gestión de los cadáveres ${ }^{5-6-7}$

Se han tenido en cuenta dos pabellones, el Palau Firal de Girona y el Pabelló Municipal Fontajau. Estos pabellones cuentan con fácil acceso, una gran capacidad de superficie, que puede albergar entre 150-200 restos cadavéricos aproximadamente, tienen refrigeración, electricidad, agua corriente y luminosidad.

Como primera opción se habilita el Palau Firal de Girona (figura1). En el caso que este estuviera ocupado por algún evento o se necesitara más capacidad por el número de víctimas, se habilitaría el Pabelló Municipal Fontajau, instalación deportiva (figura2).

Se debe recalcar en los casos de atentados terroristas la importancia de la colocación de diferentes sistemas de detección de metales (espadas y arcos) para evitar la presencia de artefactos explosivos en la zona habilitada para los trabajos de identificación.

\section{Material necesario para el proceso de identificación de los cadáveres}

El material necesario para habilitar los pabellones sería suministrado por el Instituto de Medicina Legal de Cataluña (IMLC) división Girona y la Facultad de Medicina de la Universitat de Girona (UdG).

El siguiente material daría servicio para poder gestionar entre 150-200 cadáveres y restos cadavéricos:

10 mesas de acero inoxidable $(\mathrm{UdG}) / 500$ pares de guantes de todas las medidas y tipos (IMLC/UdG)/500 batas de un sólo uso (IMLC/ UdG)/150 delantales de plástico (IMLC/UdG)/300 mascarillas de un solo uso (IMLC/UdG)/500 polainas de un solo uso (IMLC/UdG)/ 150 gorros de un sólo uso (IMLC/UdG)/10 gafas protectoras con oculares de policarbonato (IMLC/UdG)/5 ovillos de hilo de coser (IMLC/UdG)/20 agujas de coser (IMLC)/200 agujas de extracción (IMLC/UdG)/200 jeringas (IMLC/UdG)/500 tubos de muestras (IMLC/UdG)/500 etiquetas de equipaje para los cadáveres (IMLC)/500 etiquetas para el etiquetado de muestras (IMLC)/200 botes para la recogida de muestras de diferentes medidas (IMLC)/200 bisturís de un sólo uso (IMLC)/20 pinzas de disección con dientes medida estándar (IMLC/UDG)/20 
pinzas de disección sin dientes medida estándar (IMLC/UDG)/15 tijeras Mayo para cirugía (UdG)/15 tijeras universales $(\mathrm{UdG}) / 10$ pinzas hemostáticas Rochester-Pean sin dientes $(\mathrm{UdG}) / 10$ pinzas hemostáticas Rochester-Ochsner con dientes (UdG)/10 separadores de Wolkman (IMLC/UdG)/5 pinzas para huesos (IMLC/UdG)/10 costótomos (IMLC/UdG)/4 sierras de autopsia (IMLC/ UdG)/8 potes de jabón de manos (IMLC/UdG)/50 litros de desinfectante para instrumental y suelo (IMLC)/50 contenedores de residuos orgánicos de 10 litros (IMLC/UdG)/50 contenedores para basura general de 10 litros (IMLC/UdG)/150 bolsas de basura tipo II (UdG)/3 contenedores de 2 litros para todo tipo de objetos punzantes (IMLC/UdG)/6 rollos de papel industrial con sus soportes (IMLC/UdG)/250 bolsas sudario, tallas de plástico y sábanas (MÉMORA)

\section{Conclusiones}

Una vez analizados todos los medios y equipos que se disponen en una ciudad como Girona, podemos decir que la ciudad está capacitada y preparada para gestionar, actuar y acoger una catástrofe con un gran número de restos humanos, ya que dispone del material, de las infraestructuras necesarias para ser habilitadas y de un personal necesario para dar respuesta a ella, que consiste en: Médicos Forenses; Técnicos en Patología Forense; Odontólogos Forenses; Antropólogos Forenses; Personal Judicial; Policía Científica, todos ellos cualificados y experimentados para actuar.

\section{Conflicto de intereses}

No existe ningún conflicto de intereses por parte de los autores en la publicación de este artículo. 


\section{Bibliografía}

1- Barbería Marcalain E. Forensic aspects of mass fatality incidents: Barajas aircrash experience; Rev Esp Med Legal. 2009;35(1):1-2

2- Eduardo Andreu Tena, Francisco J Pera Bajo. Medical forensic performance after the Spanair airplane crash at Barajas airport, August 20th 2008. Rev Esp Med Legal. Enero2009; 35(1):12-18

3- Barbería Marcalain E, Arimany Manso J, Vidal Gutiérrez C, Bardalet Vinyals N, Hospital Ribas A, Ortigosa Ruiz JF, Aizpún de la Escosura E, Rodríguez Soto JL. Identificación de víctimas del desastre del tsunami en Tailandia. Med Clin (Barc). 2005;124: 737-41

4- Interpol. Disaster Victim Identification Guide. 2009. Chapter 5-AM Data Collection. pp 19-30

5- Interpol. Disaster Victim Identification Guide. 2009. Chapter 6- PM Evidence Collection. pp 31-41

6- Prieto JL, Tortosa C, Bedate A, Segura L, Abenza JM, Mariscal de gante MC et al. The 11 March 2004 terrorist attacks: the importance of the mortuary organization for identification of victims. A critical review. Int J Legal Med. 2007; 121: 517-22.

7- Real Decreto 32/2009, de 16 de enero, por el que se aprueba el Protocolo nacional de actuación Médico-forense y de Policía Científica en sucesos con víctimas múltiples.

8- http://www.firagirona.com

9- http://www2.girona.cat/ca/esports 


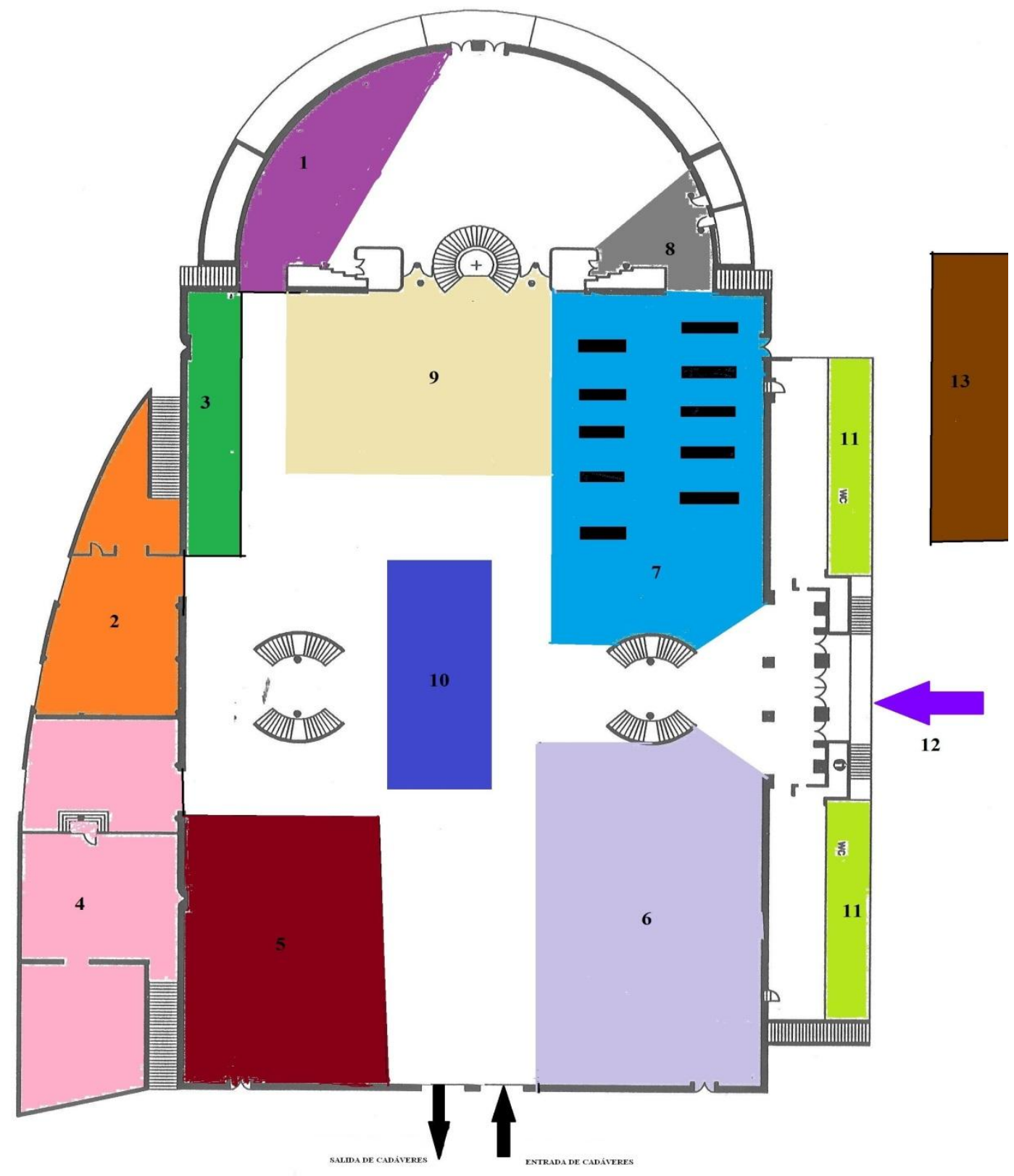

Figura1. Plano Palau Firal ${ }^{8}$ (1) Servicio de cafetería y descanso del personal. (2) Almacén. (3) Oficina Funeraria. (4) Recepción y administración. (5) Depósito de restos mortales identificados. (6) Depósito de restos mortales por identificar. (7) Área de realización de autopsias; Los rectángulos negros indican las mesas de autopsia. (8) Área de estudios complementarios. (9) Área de objetos personales. (10) Oficina judicial/Control de calidad. (11) Servicios W.C. (12) Entrada para autoridades y personal de actuación. (13) Parking para las autoridades y personal de actuación. Entre las áreas 5 y 6 se sitúa el control de ingresos de cuerpos, restos y objetos de las víctimas mortales. En estas áreas se deberían colocar detectores de metales en casos de ataques terroristas. 


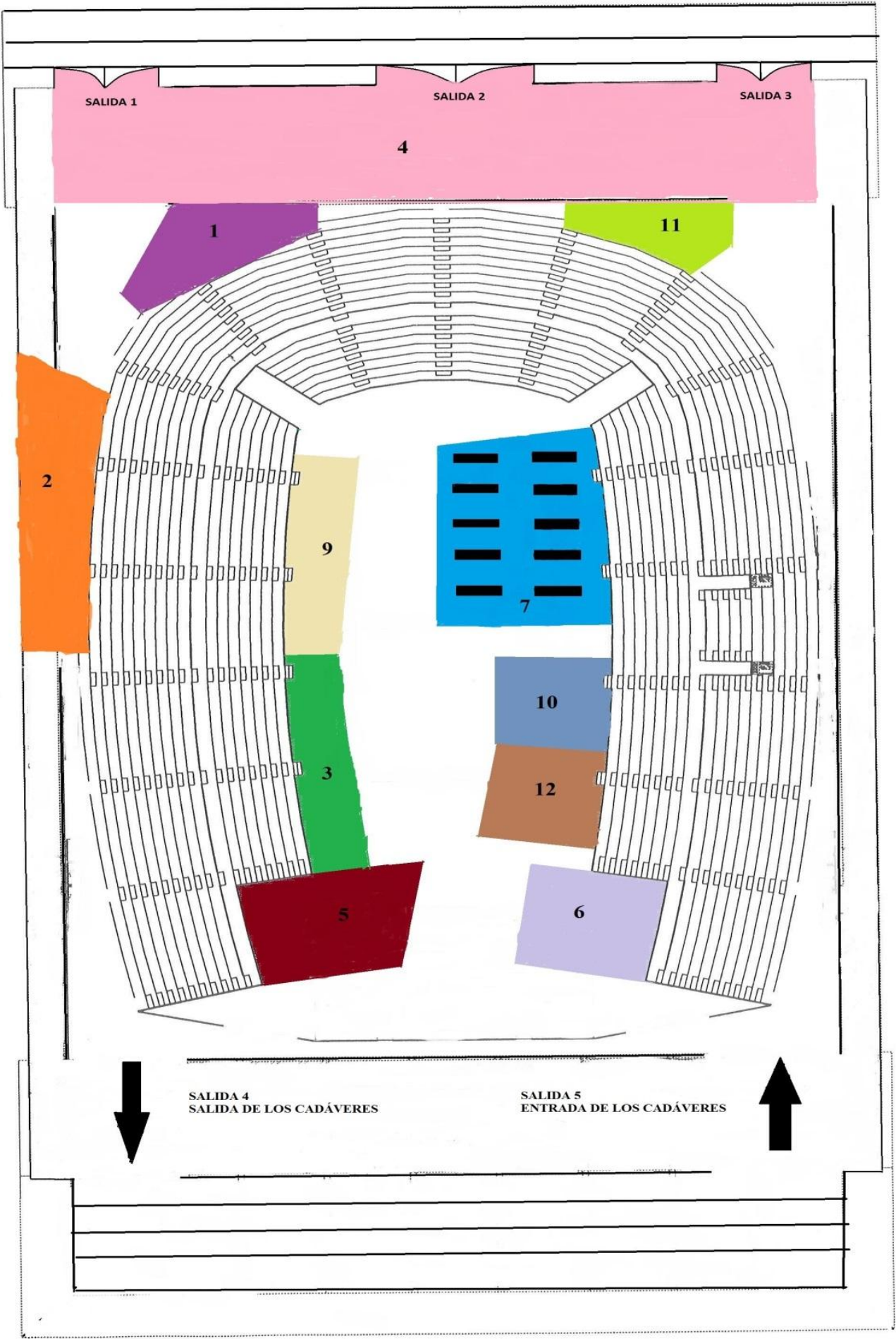

Figura 2. Plano Pabellón Municipal Fontajau9 (1) Servicio de cafetería/descanso personal. (2)Almacén. (3)Oficina Funeraria. (4)Recepción/Administración. (5) Depósito de restos mortales identificados. (6) Depósito de restos mortales por identificar. (7) Área de realización de autopsias. Los rectángulos negros indican las mesas de autopsia. (8) Área de estudios complementarios. (9) Área de objetos personales. (10) Oficina Judicial/Control de calidad. (11)Servicios W.C. (12) Control de ingresos de cuerpos, restos y objetos de las víctimas mortales. En estas áreas se deberían colocar detectores de metales en casos de ataques terroristas. 